\title{
SUSTENTABILIDAD EN LA VIVIENDA DE INTERÉS SOCIAL. Nuevas perspectivas de planeación a través de co-beneficios, análisis de caso Guadalajara-Curitiba contemporáneas
}

\author{
Dulce Esmeralda García Ruiz; Fernando Córdova Canela; Tomás Antonio Moreira \\ Universidad de Guadalajara; Instituto de Arquitetura e Urbanismo USP São Carlos \\ Director de la tesis en curso o de la investigación: Dr. Fernando Córdova Canela, UdeG \\ Co-Director de la tesis en curso o de la investigación: Dr. Tomás Antonio Moreira, IAU-USP \\ EMail: desmeralda_16@hotmail.com; fcordova.72@gmail.com; tomas_moreira@sc.usp.br
}

\section{RESUMEN}

Los procesos de transformación en la ciudad exigen un rápido atendimiento en las ciudades cada día en constante crecimiento ¿pero es posible alcanzar este crecimiento sin fin? ¿es posible dar el salto hacia un mejor futuro? Los nuevos sistemas de planeación contemporáneos en ciudades intermedias como Guadalajara y Curitiba, se han insertado bajo una dinámica en la que es posible lograr un campo de exploración de interés, al ser sede de proyectos dinámicos de importancia mundial. Al mismo tiempo de converger con grandes problemas de contaminación, desigualdad, altas demandas poblacionales, déficit de vivienda y problemáticas urbanas.

Estas ciudades al estudiarlas apuntan a ser un instrumento de apoyo para lograr la verdadera sustentabilidad a través de un enfoque multifacético y polidimensional.

Bajo el enfoque teórico y metodología sistémica de co-beneficios y análisis de caso crítico-comparativo. Este estudio puede lograr contribuir a nuevos análisis de política de vivienda en los sistemas de planeación.

Palabras clave: Sustentabilidad, vivienda social, planeación urbana, co-beneficios.

\section{ABSTRACT}

The transformational process in the city requires a fast assistance in the continuous growing cities but, is it possible to achieve this endless growth? Is it possible to step forward to a better future?

The new planning contemporary systems in intermediate cities such as Guadalajara and Curitiba, have been able to be inserted under dynamic paths that makes it possible to be a field of interest, being the headquarters of dynamic and dual projects recognized over the world. But at the same time dealing with inequality, high demand, house density, lack of housing and urban problems.

These cities aim to be a huge support instrument to achieve true sustainability through with a multifaceted and multi-dimensional approach.

Under a theoretical approach and a systematic methodology of co-benefits and critic-comparative case analysis. This study may contribute to a new analysis of housing policy in planning systems

Key words: Sustainability, social housing, Urban Planning, co-benefits 


\section{APERTURA}

Dentro de la red de posibilidades actuales en los instrumentos estratégicos de planeación urbanos y sustentables, se ha encontrado un enfoque promisorio que busca lograr llevar el planeamiento a otro nivel de desarrollo en el que con una política estratégica logre multipropósitos co-controlados en los sistemas urbanos que configuran la ciudad.

Éste enfoque busca ser una solución en una política ganar-ganar para los habitantes, el gobierno, las industrias, las sociedades civiles y el medio ambiente, las cuales exigen cada día constantes y rápidos cambios en un mundo en transformación.

Este término es denominado los co-beneficios con el que se ilustra de manera promisoria como una herramienta para lograr modelar nuevas herramientas de planeación en la ciudad, los cuales están basados en los beneficios e impactos indirectos, mostrando cómo es que una sola política o acción es capaz de lograr diversos objetivos, a través de las prácticas pre-establecidas con lo que es posible no solo incidir a un grado de actuación, si no que ésta dimensión posee multi-efectos localizados como impactos colaterales que muchas veces tienen mayor grado de incidencia en la estrategia pre-establecida y la cual configura la ciudad o medio donde se desarrolle la estrategia base localizada de forma tradicional.

Por lo que un enfoque multi-integrativo como es la sustentabilidad donde se inter-relacionan el sector económico, social y ambiental para la preservación de las generaciones futuras, debería tener también una herramienta que con una sola estrategia multi-integre varios de los efectos esperados en su desarrollo de forma localizada y medible a través de la detección de estos impactos ocultos inherentes y es por eso la importancia de estudio. Por lo que los co-beneficios si bien, apuntan para ser una herramienta viable que sirva de apoyo a la sustentabilidad de tal manera que pueda viabilizar el lograr superar la brecha de objetividad dejada por este término.

A través del planeamiento urbano con enfoque en la vivienda social, debido a la alta demanda que tienen los países latinoamericanos en satisfacer el déficit habitacional, y en la alta incidencia que tienen éstas en el sistema organizativo de las ciudades contemporáneas. Se tomará a la vivienda desde el punto de vista en que no sólo como un objeto aislado, sino que éstas pertenecen a un contexto a un conjunto urbano determinado.

Por medio de dos ciudades de importancia en Latinoamérica una considerada la más sustentable, la otra considerada la menos verde, lo cual resulte de forma complementaria, el cómo se han tomado las estrategias de planeación en miras de validar una nueva perspectiva de transformación en la ciudad y con lo cual se puedan localizar los impactos y beneficios ocultos de esta línea promisoria con la aplicación de este nuevo enfoque estratégico. Tomando el estudio bajo dos estrategias básicas de análisis para la sustentabilidad en la ciudad: la movilidad y el paisaje.

Bajo el enfoque teórico de Balaban, Smith, Thiele, Leite, ONU-Habitat, el análisis y estudio de caso y bajo la visión metodológica y prospectiva, se pretende construir y operar esta nueva perspectiva de planeamiento urbano sustentable en la vivienda de interés social a través de los co-beneficios.

\subsection{Objetivo}

Comprender la sustentabilidad en la vivienda de interés social e identificar la localización o deslocalización de los beneficios e impactos indirectos (co-beneficios) que se tienen o no en la creación y fomento de la vivienda social sustentable en los nuevos sistemas de planeación de la ciudad por medio del análisis de caso GuadalajaraCuritiba contemporáneas.

\section{MARCO TÉORICO. UNA PERSPECTIVA GLOBAL, NACIONAL Y LOCAL EN DIVERSAS REALIDADES}

\subsection{Estructura del marco teórico}

Para la estructura del marco teórico en la investigación se basó en un entendimiento del contexto del objeto de estudio correspondiente a la etapa 1 de la metodología basada en la prospectiva mediante el método del árbol 
de Marc Giget (Ver en (Gándara \& Osorio Vera, 2014) en el que se establecen tres fases generales para su abordaje: en las raíces, el tronco y el follaje.

De tal forma que la sustentabilidad y los co-beneficios se situaron como el sustento teórico base del objeto de estudio identificados como las raíces del árbol.

La política pública y los sistemas de planeación al ser los instrumentos que llevan a cabo el funcionamiento en la ciudad y la vivienda a través de objetivos, variables e indicadores, o bien las condiciones que intervienen para la implementación de las estrategias y acciones urbano-ambientales por lo que estarían situados en el nivel del tronco del árbol.

La vivienda al ser el objeto teórico y práctico que interviene en el desarrollo de esta investigación y al ser uno de los elementos básicos para la configuración de la ciudad, la disminución de la contaminación y el cambio climático a través de las estrategias y acciones de sustentabilidad para efectos de este estudio se toman como los aspectos que reflejan la parte superficial del problema o bien, misma que se abordará mediante estudio de caso para su comprobación operativa Guadalajara-Curitiba.

\subsubsection{Sustentabilidad. Enfoque urbano a través de una visión a escala global.}

Actualmente hay varios autores como Gutiérrez y González (2010), López (2014), Foladori, y Pierri, (2005) que hacen referencia a los problemas inherentes en el concepto de desarrollo sustentable anunciando que, lo que se ha llamado desarrollo es, en su expresión actual, insustentable, así como que la sustentabilidad global no puede alcanzarse extrayendo más recursos del planeta.

Para Leite y Marques, el concepto de sustentabilidad, por su carácter normativo es muy abstracto, lo que genera la necesidad de profundizar el conocimiento sobre los impactos de la actuación humana en los ambientes en que están insertados... Ellos referencian que el sector de la construcción podría estimular la sustentabilidad urbana y contribuir con la promoción del desarrollo sustentable global. Agregando que se puede alcanzar un crecimiento económico con un impacto mucho menor si pensáramos claramente, en términos de sistemas, y basados en objetivos globales.

Se ha encontrando que algunos de los ingredientes que contienen la ciudades en búsqueda de la sustentabilidad es: el adecuado y planeado uso de suelo mixto; un modelo de ciudad compacta con densidades cualificadas y multicentralidades ligadas a la red de movilidad; así como éstas se desarrollan a partir de una adecuado, amigable y ponderado enlace entre el ambiente construido y la geografía natural, con buena relación con las aguas y áreas verdes (Leite \& Marques Awad, 2014).

Así, en un intento por conocer cuáles son las ciudades más verdes de Latinoamérica, en un estudio realizado por Siemens se intentó calcular el índice de ciudades verdes en América Latina, en los resultados generales las ciudades fueron colocadas en cinco categorías de rendimiento, donde se aprecia a Guadalajara como una de las ciudades menos verdes de Latinoamérica, y a Curitiba como una ciudad muy por encima del promedio (Economic Intelligence Unit 2010. P. 9).

\begin{tabular}{|l|l|l|l|l|}
\hline $\begin{array}{l}\text { Muy por debajo } \\
\text { del promedio }\end{array}$ & $\begin{array}{l}\text { Debajo } \\
\text { promedio }\end{array}$ & Promedio & $\begin{array}{l}\text { Encima del } \\
\text { promedio }\end{array}$ & $\begin{array}{l}\text { Muy por encima } \\
\text { del promedio }\end{array}$ \\
\hline Guadalajara & Buenos Aires & Medellín & Belo Horizonte & Curitiba \\
\hline Lima & Montevideo & Ciudad de México & Bogotá \\
\hline & & Monterrey & Brasilia & \\
\hline & & Porto Alegre & Rio de Janeiro & \\
\hline & Puebla & São Paulo & \\
\hline & Quito & & \\
\hline & & Santiago & & \\
\hline
\end{tabular}

Tab.0. Índice de ciudades verdes en América Latina, resultados generales; las ciudades fueron colocadas en cinco categorías de rendimiento que son los porcentajes fuera de la media y los que están encima de la media. (Economic Intelligence Unit 2010, 9). 
Estas dos ciudades también son catalogadas bajo la categoría de ciudades intermedias, debido a su escala, crecimiento poblacional y crecimiento económico sostenido como se puede localizar en el siguiente mapa (Banco Interamericano de Desarrollo, 2014; The economist, 2015).

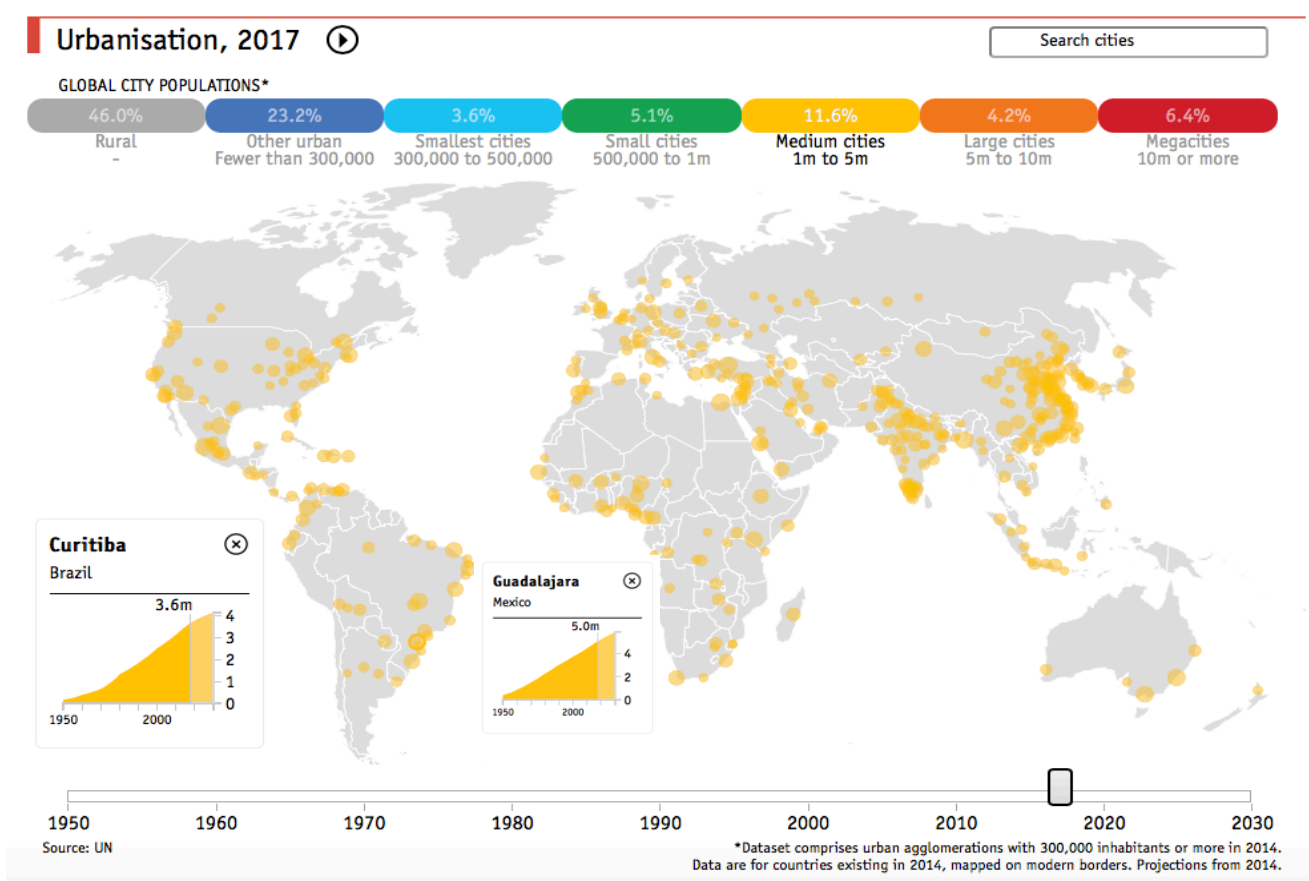

Ciudades intermedias a nivel mundial. Urbanización 2017 Guadalajara-Curitiba. (The economist, 2015)

Bajo este esquema también se pretende constatar si realmente hay una brecha metodológica dejada y muchas veces criticada por el término de sustentabilidad a través del estudio de casos. Y con lo cual mediante ésta nueva estrategia de los co-beneficios pueda ayudar a identificar y disminuir el sesgo de operatividad y objetividad dejada por este concepto, a través de instrumentos alternativos, y con ayuda del análisis del estudio de caso de dos ciudades de características similares (en cuestión de escala, habitantes y pertenecientes a dos de las economías más importantes de Latinoamérica) lo cual demuestre de manera tangible la importancia del estudio.

\subsubsection{Co-beneficios y vivienda. En búsqueda de la relación conceptual}

El término de co-beneficios aparecio en la literatura académica en los años 90 , generando un mayor interés en la época del Tercer Informe de Evaluación (AR3), sin embargo es hasta 2002, cuando se publicó oficialmente este enfoque a través del Panel Intergubernamental de Cambio Climático (IPCC).

Para el US EPA, Observatorio de Manila y CAI-Asia el concepto de una "estrategia de co-beneficios" o una "medida de co-control" indica que se trata de una sola actividad o política que puede generar múltiples beneficios a través de varios sectores o campos de estudio. A esto IPCC argumenta y diferencia a los "co-beneficios" como beneficios esperados del objetivo principal de ciertas acciones o políticas de las que no lo son. (Extraído de (García, 2016).

Por su parte Smith, aborda en su libro "El bono climático: los co-beneficios de la política climática" en el que explica que los co-beneficios son los beneficios adicionales que obtenemos cuando actuamos para controlar el cambio climático, por encima y más allá de los beneficios directos. Ellos se refieren a veces como "múltiples beneficios" de "sinergias" (Smith, A. 2013).

Mismo que para el objeto de estudio se va a referir al concepto de los co-beneficios definidos como una decisión intencional con respecto en una concepción híbrida destinada a objetivos específicos en diferentes áreas con estrategias de co-control que disminuyen el calentamiento global, la contaminación, la conservación de los 
recursos vitales como energía, la comida y que además considera otros beneficios específicos de gran relevancia como la mejora de las viviendas y la planificación urbana, reducción de costos y retorno de períodos de capital y mantenimiento, reducción de impactos en la salud, la mejora de la economía. Bajo la implementación de políticas públicas, centradas en un enfoque "ganar-ganar "entre la naturaleza, la sociedad, el gobierno, las sociedades civiles y las industrias con lo que significaría diversos impactos adicionales en beneficio de todos con medidas de intervención de tiempo en diferentes escalas (García, 2015).

Dividiéndolos en seis categorías basadas en Smith (2013): Aire limpio; Tierra más verde; Energía segura; Menos residuos; Economía más fuerte; Salud y bienestar.

\subsubsection{Vivienda de interés social un paso para la sustentabilidad.}

Para Abiko, la vivienda de interés social, es un término genérico que define una determinada solución de la vivienda enfocada para la población de bajos ingresos. Donde la vivienda para la población de bajos ingresos (housing for low income people) lleva la necesidad de definir el salario máximo y mínimo de las familias, misma que está inmersa en las condicionantes de la actual política pública tanto en México como en Brasil. A esto Abiko (1995) también agrega, que la vivienda popular no debe ser entendida como un producto y si como un proceso, con una dimensión física pero también como resultado de un proceso complejo de producción con determinantes político, sociales económicos, jurídicos, ecológicos, tecnológicos (Abiko, 1995).

Debido a la importancia que tiene la vivienda desde un punto básico de dar protección, como agente transformador de la cultura de un pueblo, la reducción de pobreza, estabilidad y vulnerabilidad social, así como se trata también de una medida detonadora del crecimiento económico, al mismo tiempo que en la postura ambiental es responsable de grandes cantidades de emisiones y residuos, lo cual involucra un potencial de reducción. La importancia del abordaje de la vivienda para este estudio como agente detonante se puede diagramar en la siguiente figura:

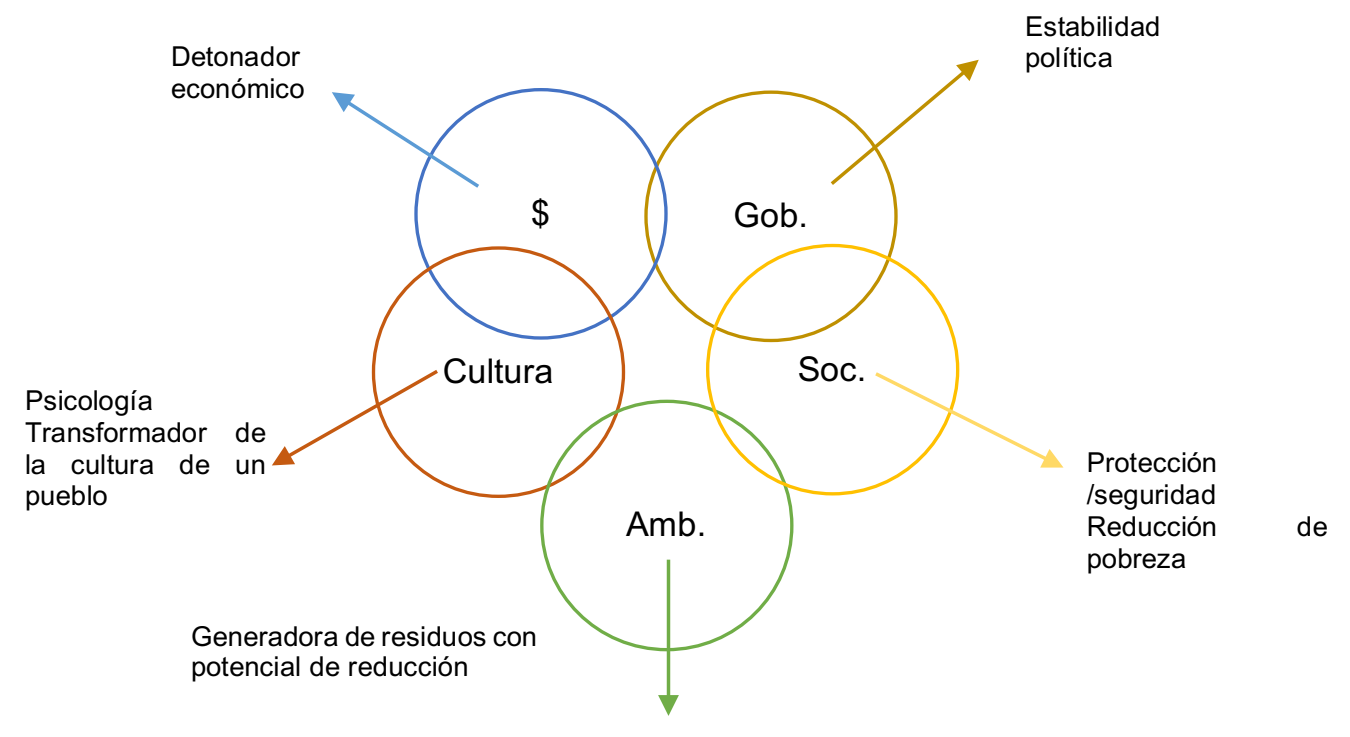

Diagrama de componentes de la vivienda. Elaboración propia.

Mismo que ONU-Habitat referencía a "las viviendas sostenibles como aquellas que pueden ser tanto inclusivas como accesibles para todos. Sin embargo, ser asequible no es la única condición, ya que este tipo de viviendas no pueden considerarse sostenibles si generan un impacto negativo en el medio ambiente o en la vida social. Así pues, solo la unión entre viviendas asequibles y las demás condiciones de sostenibilidad garantizan el verdadero significado de una vivienda sostenible. Adicionalmente, aunque una vivienda sostenible también es considerada una vivienda verde, es necesario adoptar una perspectiva y un enfoque más amplio que permita entender a una vivienda sostenible no solo como una unidad o un grupo de edificios verdes auto-sostenibles, sino como una 
práctica residencial, amigable con el ambiente y con la sociedad que se integra con los sistemas urbanos y/o asentamientos" (ONU HABITAT, 2016).

Actualmente, a pesar de los avances que la cuestión en la vivienda social ha alcanzado en la agenda política. Varios autores agregan que todavía no existe una gran ruta en busca de una vivienda más sustentable y sistémica, sobre todo, en el ámbito de arquitectura y urbanismo, para su rápida implementación.

\subsection{Política pública y sistemas de planeación y sus Implicaciones en el estudio.}

Actualmente la política pública tradicional se ha llevado desde una perspectiva en la que ante una problemática existe una estrategia para controlarlo, con lo que se obtiene un beneficio directo prestablecido (ahorro de costo, satisfacción de demanda, fomento económico, reducción de emisiones) sin embargo, si se lleva y analiza esto al ámbito urbano, en un segundo o hasta tercer nivel de análisis es posible detectar que dicha estrategia direccional, deriva en diversos impactos ocultos inherentes como expansión de la ciudad, aumento de contaminación, degradación de las reservas ambientales, aumento de servicios derivados de la expansión urbana, lo que por ende se deriva en una mayor problemática urbana, social, económica y ambiental.

Del tal forma que al ser los co-beneficios una categoría conceptual y analítica que se trata de implementar a nivel política pública en los sistemas de planeación de la ciudad y la vivienda, para la localización de los impactos y beneficios ocultos de forma clara, objetiva y sistémica, es necesario abordar a esta misma para comprender su relación conceptual. Por una parte, es necesario identificar el problema público a través del diagnóstico del mismo y por otro lado la política pública, la cual como afirma Secchi, L. (2016) es el medio o mecanismo para llevar a cabo la intención, a través instrumentos concretos como programas y planes, etc.

Por lo que para este estudio se tomará como referencia el abordaje de la política pública relevante al ámbito de vivienda en diversas escalas de actuación donde se identifique la problemática a través de sus instrumentos de planeación, por lo que primero se abordará la escala bi-nacional México -Brasil; luego a escala local GuadalajaraCuritiba así como con su relación con su la región metropolitana para su comprobación operativa insmersos en sus sistemas de planeación.

\subsubsection{Contexto actual de la política pública México-Brasil que tiene influencia en el sistema de planeación en la ciudad y vivienda. Una visión a escalas nacionales}

Para el abordaje de los co-beneficios en su insignia metodológica perceptual, es necesario poder entender el contexto de la política pública donde se pretenda hacer el abordaje del objeto de estudio en este caso tomando como referencia clave las dos potencias latinoamercianas México-Brasil, además, si bien el periodo de estudio en el que se ubicará tomando como un época de mayor referencia es a partir del 2012 hasta el 2016.

La preocupación por atender en México tanto el rezago de vivienda, como el climático a través de los objetivos de la sustentabilidad puede localizarse con mayor énfasis a partir de 2012, al publicarse la Ley General de Cambio Climático, a partir de esta etapa, se establecen las nuevas Reglas de Operación para adoptar el nuevo Modelo de Desarrollo Urbano y Vivienda, mismo que toma forma más sólida al oficializarse las Acciones de Mitigación Nacionalmente Apropiadas o NAMAs de Vivienda Sustentable en ese mismo año. A partir de este período se crean las primeras viviendas piloto construidas bajo el esquema NAMA en Hermosillo, Morelia y Guadalajara (NAMA de Vivienda, 2016) en 2014 se oficializa el Facility Fund del NAMA, así como la NAMA Urbana, que será parte del esquema de implementación para la construcción de vivenda sustentable en México.

Así pues en México, se tiene un esquema de planeación a través de diversos ejes. Este sistema de planeación es de reciente creación e implementación, lo que resulta de un escenario propicio para el estudio y adopción de nuevas estrategias de planeación que promuevan un enfoque multifacético e integral con una visión para lograr una coevolución urbana sistémica en la ciudad y los subsistemas que la conforman, por lo que por otro lado el abordaje del estudio de caso Brasileiro resulta complementario para el estudio de forma objetiva ya que contempla un planeamiento urbano de forma histórica como referencia mundial en algunas de sus ciudades como es el caso de la ciudad de Curitiba, Paraná.

Para el caso de la vivienda particularmente dentro de este ámbito en Brasil, destaca el sistema de certificación ambiental para construcciones sustentables Selo Casa Azul CEF por ser el primer sistema de clasificación de la 
sustentabilidad de proyectos ofertado en Brasil, desarrollado para la realidad de la construcción habitacional brasileira (Fortunato, R. A, 2014), este criterio de evaluación es de carácter voluntario y es complementario a la política pública actual implementada en los proyectos del Programa Minha Casa Minha Vida en Brasil, con los que buscan lograr la sustentabilidad en las unidades habitacionales de interés social, y el cual se tomará como referencia para el abordaje en este estudio.

\subsection{Sistemas de planeación y política pública en la vivienda social a través del estudio de caso Guadalajara-Curitiba. Diversas visiones a escala local para su comprobación operativa.}

De acuerdo con el censo de Población y Vivienda entre 2000 y 2010, en el Área Metropolitana de Guadalajara (AMG) hubo un crecimiento de 9,376 nuevos domicilios. Lo que en base a los datos censales ponen en evidencia que existe un fuerte crecimiento poblacional, pero sobre todo en la periferia metropolitana. Aunado a esto según estimaciones a través del SNIIV 2.0 en ésta área hay un rezago de 317,970 viviendas dentro de las cuales 137,970 corresponden al esquema de vivienda económica de hasta 2.6 VSM (Valor salario mínimo), siendo Guadalajara, Zapopan, Tlaquepaque, y Tonalá los que requieren la mayor demanda por ésta misma (Demanda Potencial INFONAVIT, 2016).

En el caso brasilerio, de acuerdo con el censo de 2010, Curitiba posee 650,620 domicilios. Entre 2000 y 2010 hubo un crecimiento de 156,290 nuevos domicilios. El 40\% de este crecimiento se concentró en los barrios perifericos con la región metropolitana. De acuerdo con el salario domiciliar el $28 \%$ de estos domicilios se encuentran en el rango de hasta 3 salarios mínimos, $21 \%$ entre 3 y 5 salários minimos, $27 \%$ entre 5 y 10 salários minímos y $24 \%$ encima de 10 salários minimos (IPPUC, 2014)

\subsubsection{Programas, políticas y planes en la vivienda social Guadalajara-Curitiba. Contexto y panorama actual}

Dentro de este panorama actual de política pública de vivienda en Guadalajara, Jalisco, México, destaca el programa NAMA de Vivienda desarrollado mediante la colaboración mexicano-alemana, a través de la Comisión Nacional de Vivienda (CONAVI). En donde como parte de las acciones establecidas de este programa se realizaron diversos prototipos en el caso de Guadalajara, fuero unidades habitacionales de vivienda adosada en la categoría vivienda social-popular de hasta 4 salarios mínimos, con la tipología vertical llevando a cabo 18 edificios de 3 niveles, en un área de $4767 \mathrm{~m} 2$, en el municipio de Tonalá, Jalisco, dentro del Área Metropolitana de Guadalajara, buscando lograr escalar la eficiencia hasta $60 \%$ respecto a cómo se construía con el programa anterior de hipoteca verde (NAMA de Vivienda Nueva, 2016).

En el caso de Curitiba, Paraná, Brasil, a partir del 2009, el Gobierno Federal marca como prioritario el lanzamiento del Programa Minha Casa Minha Vida (PMCMV) donde busca disponibilizar el crédito y subcidios para adquisición de vivienda de interés social para las familias de hasta tres salarios mínimos (Ministerio das Cidades, 2016). Dentro del este programa se manejan 4 modalidades de créditos y subsídios que van desde la faixa 1, para familias con un ingreso mensual de hasta 1.800,00 reales y donde tiene una capacidad de subsídio de hasta $90 \%$ del valor del inmueble, el pago es hasta en 120 prestaciones, sin intereses y hasta la faixa 3, para familias con un ingreso mensual de hasta $6.500,00$ reales en la modalidad de financiamiento con un interés de $8.16 \%$ de interés por año. Por lo que dependiendo del ingreso mensual mínimo es asignado el recurso para el programa (Ministerio das Cidades, 2016; Esmanhotto C, 2016), dentro de los cuales es posible localizar diversos conjuntos habitacionales de estas características, realizados en Curitiba y su región metropolitana.

\subsubsection{Plan director Guadalajara-Curitiba}

El plan director es uno de los instrumentos principales de la política pública a la hora de planear y dirigir la ciudad, de tal forma que sea posible llevar a cabo las estrategias clave para su implementación dentro de la ciudad, como dice Miguel Roguski del Instituto de Pesquisa e Planejamento Urbano de Curitiba (IPPUC) el plan director es el futuro del funcionamiento de una ciudad (Roguski, 2016).

En lo que respecta en Guadalajara, no se ha contando con un principio rector que direccione, estructure y proponga el crecimiento planificado histórico a través de la sustentabilidad, tanto a nivel ciudad como con su región metropolitana, más sin embargo en 2016, a través del IMEPLAN (Instituto Metropolitano de Planeación)se ha publicado en junio lo que serían las directrices para orientar el planeamiento en el AMG a través del POTmet 
o bien, Programa de Ordenamiento Territorial metropolitano, el cual ya cuenta con una visión a largo plazo de planeamiento para Guadalajara 2042, como estrategias de redensificación en el área central de la metrópoli, atender la demanda de vivienda; así como considerar la gestión ambiental metropolitana (IMEPLAN, 2016), por lo que resulta ser punto clave para la generación de nuevas estrategias que transiten hacia la coevolución urbana sustentable sistémica como referencia nacional.

En el caso de Curitiba el plan director si bien resulta de interés para su consulta y estudio ya que es interesante poder obtener datos que puedan validarse a través del comportamiento de los indicadores en el tiempo a través de estos dos estudios de caso diversos, donde en Curitiba aún puede verse en la ciudad las directrices que lo conformaron desde 1966 por medio de estrategias de sustentabilidad que marcaron este periodo a través del mandato de Jaime Lerner, priorizando la conformación linear de la ciudad con la implantación de los ejes de uso mixto y transporte público. Así como las estrategias adoptadas para el paisaje urbano. Actualmente también se han previsto de nuevas estrategias que ayuden a disminuir la actual problemática del rezago habitacional optando por estrategias integradoras en las que los nuevos desarrollos urbanos adopten y consideren un porcentaje para vivienda de interés social dentro del mismos (IPPUC, 2014).

Realizando una síntesis del abordaje de estas dos ciudades latinoamericanas presentamos la siguiente tabla:

\begin{tabular}{|l|l|}
\hline Guadalajara (AMG) & Curitiba (RMC) \\
\hline $\begin{array}{l}\text { Considerada una de las ciudades menos verdes } \\
\text { en Latinoamérica (Siemens, 2010) }\end{array}$ & $\begin{array}{l}\text { Considerada la ciudad más verde de } \\
\text { Latinoamérica (Siemens, 2010) }\end{array}$ \\
\hline $\begin{array}{l}1495189 \text { millones de habitantes en la ciudad } \\
\text { central (INEGI, 2010) }\end{array}$ & $\begin{array}{l}1751 \text { 907 millones de habitantes en la } \\
\text { ciudad central (IBGE, 2010) }\end{array}$ \\
\hline Por su escala es ciudad intermedia & Por su escala es ciudad intermedia \\
\hline $\begin{array}{l}4434878 \text { habitantes en el Área Metropolitana de } \\
\text { Guadalajara (INEGI, 2010) }\end{array}$ & $\begin{array}{l}3174201 \text { habitantes en la Región } \\
\text { Metropolitana de Curitiba (IBGE, 2010) }\end{array}$ \\
\hline $\begin{array}{l}\text { Es la segunda concentración poblacional, } \\
\text { económica y territorial más importante de México }\end{array}$ & $\begin{array}{l}\text { Es la cuarta aglomeración urbana con } \\
\text { mayor Producto Interno Bruto en Brasil. }\end{array}$ \\
\hline $\begin{array}{l}\text { Alberga proyectos piloto de NAMA de vivienda, } \\
\text { correspondientes a la política actual } \\
\text { implementada en México con respecto a la la } \\
\text { vivienda de interés social. }\end{array}$ & $\begin{array}{l}\text { Alberga proyectos de PMCMV de los tres } \\
\text { tipos de faixas con respecto a los programas } \\
\text { federales de la política pública actual } \\
\text { implementada en Brasil con respecto a la } \\
\text { vivienda de interés social. }\end{array}$ \\
\hline $\begin{array}{l}\text { No cuenta con un historial de planeamiento } \\
\text { sustentable. }\end{array}$ & $\begin{array}{l}\text { Cuenta con un historial urbano de } \\
\text { planeamiento sustentable a largo plazo aún } \\
\text { tangible en la ciudad. }\end{array}$ \\
\hline \begin{tabular}{l}
51 m2 de área verde por habitante \\
\hline
\end{tabular}
\end{tabular}

Tabla 1. Síntesis del abordaje comparativo general entre Guadalajara y Curitiba con aspectos relevantes urbanos y habitacionales. Elaboración propia a partir de varias fuentes.

\section{HIPÓTESIS}

La herramienta de los co-beneficos viabilizan la localización y deslocalización de los beneficios e impactos indirectos en los sistemas de planeación urbana y habitacional de la ciudad, que tienen incidencia en los planes y políticas municipales y federales de la vivienda de interés social, de tal manera que mediante este enfoque se puedan establecer nuevas perspectivas de planeación a través de estrategias poli-integrativas más claras, viables y accesibles para el logro de los objetivos finales en diversas escalas que se persigan a través de la sustentabilidad.

\section{METOdOLOGÍA}

Una vez entendidos los conceptos clave para el abordaje de estudio se tiene que para la implementación metodológica será necesario del método sistémico, por lo que dicho estudio se compone en dos etapas clave. 
La primera etapa de una forma exploratoria aludió al entendimiento del contexto, a través de fuentes primarias y secundarias, con lo que se obtengan diversos puntos críticos comparativos y se puedan definir y contrastar las variables más importantes como punto de partida y toma de referencia para el estudio de caso.

La segunda etapa constituyó el estudio y análisis de caso, esta etapa es clave para poder visualizar, localizar o deslocalizar los beneficios o impactos indirectos a través de los sistemas de planeación de la actualidad, a través de la correlación de datos de los estudios de caso para su comprobación operativa.

Tomando como punto de referencia el estudio de Córdova, 2015, para el caso en México, en el cual identifica cómo es concebida la sustentabilidad en los proyectos de arquitectura para la vivienda de interés social en Guadalajara implementados a través de los sistemas de planeación del Gobierno Federal. Así como a través del estudio previo de Fortunato, 2014, en el cual este estudio identifica cómo es concebida la sustentabilidad en los proyectos de arquitectura para la vivienda de interés social en Curitiba implementados a través de los sistemas de planeación del Gobierno Federal en Brasil desarrollado a través de la metodología selo casa azul.

A través de estos estudios previos se puede lograr traducir este un segundo y hasta tercer nivel de análisis que tiene como implicación los beneficios e impactos indirectos a través del sistema metodológico construido por el enfoque de los co-beneficios para la vivienda de interés social.

Mismo que pueda contribuir para futuros análisis de la política de vivienda social y a la toma de decisiones en relación a la política, estrategias, planes y métodos a través de la introducción de los indicadores indirectos en los sistemas de planeación que son el presente y futuro clave para las ciudades en México, Brasil y contextos de ciudades afines en el mundo.

\subsection{Desarrollo de la metodología basada en co-beneficios a través de los estudios de caso Guadalajara- Curitiba}

Guadalajara tiene una población de 1,495 millones de habitantes (INEGI, 2010). Esta conurbación es la segunda concentración poblacional, económica y territorial más importante de México. Para el abordaje del estudio también es de interés en esta área ya que según Siemes, catalogó a Guadalajara como la ciudad menos verde de Latinoamerica.

Curitiba es sede de proyectos para el abordaje sustentable como referencia mundial. Corresponde según Siemens como la ciudad más verde en Lationoamérica. Su población es de aproximadamente 1,751 millones de habitantes lo que la hace estar dentro de la cualidad de ciudad intermedia, sobre sale por tener $51 \mathrm{~m} 2$ de área verde por habitante y una red integrada de transporte público, lo que la hace tener ingredientes clave para considerarla ciudad sustentable.

Para poder entender los efectos en la planeación de la ciudad, es necesario de un enfoque que sea capaz de visualizar diversas escalas de actuación, así como los diversos subsistemas en que se compone por lo que el desarrollo de la metodología está basado en la localización de los co-beneficios que deberían ser atributo base para lograr cualquier giro en torno a un desarrollo urbano más sustentable. Así pues, en la primer fase para el desarrollo metodológico se hace referencia al instrumento de "Diagrama de flujo general para la metodología de evaluación de co-beneficios en la vivienda a estudiar" que establece los puntos clave del instrumentario metodológico para la evaluación por medio de este enfoque y el cual se muestra a continuación en la tabla 2, tomando en cuenta que este instrumentario brinda el camino base del estudio. Dentro del cual para la aplicación metodológica el primer paso es documentar los datos generales del estudio de caso, por lo que se completa la ficha técnica a través de la siguiente Tabla 1. 


\begin{tabular}{|c|c|c|c|c|c|c|}
\hline $\begin{array}{l}\text { Nombre } \\
\text { vivienda } \\
\text { conjunto } \\
\text { urbano }\end{array}$ & $\begin{array}{l}\text { Estado de } \\
\text { ocupación }\end{array}$ & $\begin{array}{l}\text { Estado de } \\
\text { calidad } \\
\text { (sustentable } \\
\text { o no } \\
\text { sustentable) }\end{array}$ & Total m2 & Localización & Otros & $\begin{array}{lc}\text { Número } & \text { de Número de } \\
\text { Unidades } & \text { personas } \\
\text { Habitacionales proyectadas }\end{array}$ \\
\hline $\begin{array}{l}\text { A.Nama } \\
\text { Vivienda } \\
\text { Sustentable, } \\
\text { Rinconada } \\
\text { del Rosario }\end{array}$ & Ocupado & $\begin{array}{l}\text { Sustentable } \\
\text { clasificación } \\
\text { NAMA }\end{array}$ & $\begin{array}{l}\text { Aprox. } \\
\text { 4767M2 }\end{array}$ & $\begin{array}{l}\text { Tonalá, } \\
\text { Jalisco, } \\
\text { México } \\
\text { AMG }\end{array}$ & \begin{tabular}{lr}
\multicolumn{2}{|c}{ Previo estudio con } \\
sistema & de \\
indicadores & por \\
Laboratorio & \\
Nacional & de \\
Vivienda UDG & \\
\end{tabular} & $\begin{array}{l}18 \text { unidades } 70 \\
\text { habitacionales } \\
\text { en } 3 \text { edificios } \\
\text { de } 3 \text { niveles }\end{array}$ \\
\hline $\begin{array}{l}\text { A.PMCMV } \\
\text { Conjunto } \\
\text { Hab. Parque } \\
\text { I, II, III }\end{array}$ & Ocupado & $\begin{array}{l}\text { No } \\
\text { Sustentable } \\
\text { clasificación } \\
\text { PMCMV }\end{array}$ & $\begin{array}{l}\text { Aprox. } \\
130,000 \mathrm{~m} 2\end{array}$ & $\begin{array}{l}\text { Curitiba, } \\
\text { Paraná, } \\
\text { Brasil } \\
\text { AMC }\end{array}$ & $\begin{array}{l}\text { Previo estudio con } \\
\text { sistema de } \\
\text { certificación de } \\
\text { Sustentabilidad } \\
\text { Casa azul }\end{array}$ & $\begin{array}{l}3217 \text { (ya11,178 ya } \\
\text { estaban ahí). vivían ahí } \\
2796 \text { nuevas personas. } \\
\text { unidades Hab. } 9700+1400 \\
+287 \text { PAC PAC }\end{array}$ \\
\hline
\end{tabular}

Tabla 1: Tabla ficha técnica con la tabla de identificación de edificación. Elaboración propia a partir de datos de estudios previos (Cordova, 2015; Fortunato, 2014)

\begin{tabular}{|c|c|c|}
\hline \multicolumn{3}{|c|}{ 1. Identificación de los co-beneficios la condición es que: } \\
\hline a & a. Puedan ser medidos con alguna de la metodologías. & \\
\hline & Sean alcanzables al corto plazo. & $\mathrm{x}$ \\
\hline & Sean económicamente viables & $\mathrm{x}$ \\
\hline & $\begin{array}{l}\text { Dicha decisión intencional indirecta deberá poder comprobar al menos } 3 \text { co-beneficios qu } \\
\text { de cómo resultado de su acción. }\end{array}$ & \\
\hline \multicolumn{3}{|c|}{ 2.Evaluación de los co-beneficios: } \\
\hline \multicolumn{3}{|c|}{$\begin{array}{l}\text { a. Selección de las categorías de evaluación (I, II, III, IV, V, VI) } \\
\text { (1 refiérase a calidad del aire) }\end{array}$} \\
\hline & $\begin{array}{l}\text { Selección de las subcategorías de co-beneficios: I. A.b.c. } \\
\text { A. Reducción en } \mathrm{CO} 2 \text { por el uso de Transporte Público proyectado. } \\
\text { B. Reducción del } \mathrm{CO} 2 \text { en áreas urbanas por el uso de infraestructuras de los servicio } \\
\text { básicos colocado a distancias de proximidad. } \\
\text { C. Reducción en emisiones de } \mathrm{CO} 2 \text { por la inserción en la mancha urbana }\end{array}$ & $\begin{array}{l}\text { A. } \\
\text { B. } \\
\text { C. }\end{array}$ \\
\hline & C. c. Selección del nivel del estudio que se tomará si está a nivel: & \\
\hline & $\begin{array}{l}\text { i. Plan, política, reglamento, proyecto. } \\
\text { ii. Si ya es un proyecto realizado } \\
\text { Monitoreo }\end{array}$ & \\
\hline & $\begin{array}{l}\text { Cálculo de la evaluación (ecuación definida en términos de teoría de conjuntos } \\
\text { base y proyectado) o bien en base a tabla si es cualitativo. } \\
\text { (B.1.) Primera fase experimental metodológica. }\end{array}$ & \\
\hline
\end{tabular}

Tabla 2: Diagrama de flujo general para la metodología de evaluación de co-beneficios en la vivienda a emplear. Elaboración propia a partir de datos de estudios previos

Basados en el estudio previo del Laboratorio Nacional de Vivienda y Comunidades Sustentables coordinado por Córdova 2015, para medir la sustentabilidad a través de su sistema de indicadores en el estudio de caso del conjunto urbano y habitacional "Rinconada del Rosario" Tonalá, Jalisco, dentro del AMG, y basados en la metodología de los co-beneficios se estará analizando tres indicadores que tienen como base en la medición de la sustentabilidad del estudio previo teniendo el 1.1.Localización e integración de la acción urbanística (lau); 1.2. Conectividad no motorizada de la acción urbanística; 1.3 Proximidad a las paradas de transporte público de la acción urbanística.

Así mismo basados en el estudio previo de Fortunato, (2014), para medir la sustentabilidad a través de la certificación brasileña "selo Casa Azul" en el estudio de caso del conjunto habitacional "Parque Iguaçu I, II y III, del barrio de Guanchinho, Curitiba, Paraná, (1), y basados en la metodología de los co-beneficios se estará analizando la estrategia de 1.2.Calidad Urbana dentro de la vertiente de las estrategias de esta certificación en materia de sustentabilidad,

Para ambos casos se aplicará basado en la metodología de lo co-beneficios, la categoría I Calidad del Aire y como sub-categorías de su validación se tomará A. Reducción en $\mathrm{CO} 2$ por el uso de Transporte Público proyectado; $\mathrm{B}$. Reducción del $\mathrm{CO} 2$ en áreas urbanas por el uso de infraestructuras de los servicios básicos colocado a distancias de proximidad y C. Reducción en emisiones de CO2 por la inserción en la mancha urbana como objeto de estudio, tal y como se muestran en la tabla 3. Y de lo cual debería ser parte básica de la estrategia de planeación en miras de lograr los objetivos con respecto al cambio climático. 


\begin{tabular}{|c|c|}
\hline \multicolumn{2}{|c|}{$\begin{array}{l}\text { Estrategia general sustentabilidad en base a indicadores de Sustentabilidad } \\
\text { de Vivienda y Comunidades Sustentables (Córdova, 2015): } \\
\text { 1.1. Localización e integración de la acción urbanística (lau) } \\
\text { 1.2. Conectividad no motorizada urbanística } \\
\text { 1.3. Proximidad a las paradas de transporte público de la acción urbanística } \\
\text { Estrategia general sustentabilidad (Fortunato, 2014): } \\
\text { 1.2 Calidad Urbana- Certificación Selo Casa Azul }\end{array}$} \\
\hline \multirow{4}{*}{$\begin{array}{l}\text { Categoría general I de los } \\
\text { co-beneficios. } \\
\text { I. Aire limpio }\end{array}$} & Sub-categoría o variables de co-beneficios: \\
\hline & A. Reducción en CO2 por el uso de Transporte Público proyectado. \\
\hline & $\begin{array}{l}\text { B. Reducción del CO2 en áreas urbanas por el uso de infraestructuras de } \\
\text { los servicios básicos colocado a distancias de proximidad. }\end{array}$ \\
\hline & C. Reducción en emisiones de $\mathrm{CO} 2$ por la \\
\hline
\end{tabular}

Tabla 3: Elección de categorías de análisis a estudiar previo a la evaluación de la sustentabilidad y en miras de localizar los cobeneficios o co-impactos que producen las estrategias implementadas. Elaboración propia

Una vez atendiendo este primer recurso identificado, se localiza qué nivel metodológico se utilizará teniendo en cuenta lo siguiente basado en (Manual for Quantitative evaluation of the co-benefits approach to climate change projects, 2009) en donde se establecen 3 niveles de evaluación, el nivel 1 el más sencillo cuando se tiene dificultad de formular las ecuaciones necesarias derivadas de un cálculo cualitativo; el nivel 2 conduce a utilizar una ecuación predeterminada y el nivel 3 también corresponde a una evaluación cuantitativa pero con datos de ecuaciones específicas.

Para el desarrollo de este estudio se estará tomando los niveles 1 y 2 (ver tablas 4 y 5 ), con lo que se pueda contrastar los resultados de la implementación metodológica de estos dos niveles y ver si existiera o difirieran resultados.

\begin{tabular}{|c|c|c|c|c|c|c|c|}
\hline $\begin{array}{l}\text { Categoría } \\
\text { Principal en } \\
\text { referencia a la } \\
\text { Sustentabilidad) }\end{array}$ & $\begin{array}{l}\text { Categoría } \\
\text { de } \\
\text { Evaluación } \\
\text { (co- } \\
\text { beneficio) } \\
\end{array}$ & $\begin{array}{l}\text { Tipo: } \\
\text { Plan, } \\
\text { aplicación... }\end{array}$ & $\begin{array}{l}\text { Criterio } \\
\text { aplicado }\end{array}$ & Variables estratégicas & $\begin{array}{l}\text { Indicador: } \\
\text { Reducción de } \\
\text { emisiones } \\
\text { esperadas en } \\
\text { kgCO2e }\end{array}$ & $\begin{array}{l}\text { Resultado } \\
\text { caso } \\
\text { Guadalajara }\end{array}$ & $\begin{array}{l}\text { Resultado } \\
\text { caso } \\
\text { Curitiba }\end{array}$ \\
\hline Calidad urbana & Aire limpio & Aplicación & A. & $\begin{array}{l}\text { Identificación de los } \\
\text { servicios de TP con sus } \\
\text { respectivas distancias. } \\
\text { Escenario base } \\
\text { cumplimiento con } 1 \\
\text { parada ya ubicada } \\
\text { anterior al desarrollo. } \\
\text { Escenario proyectado } \\
\text { por estrategias de } \\
\text { sust=2 Paradas de TP, } \\
\text { para acciones urbanas } \\
\text { de menor o igual a } \\
16 \text { ha. }\end{array}$ & $\begin{array}{l}\text { Por cada } 1000 \\
\text { km, reducir } 767 \\
\text { CO2 kg de } \\
\text { CO2e }\end{array}$ & $\begin{array}{l}25 \% \text { de lo } \\
\text { esperado }\end{array}$ & $\begin{array}{l}50 \% \text { de lo } \\
\text { esperado }\end{array}$ \\
\hline Calidad urbana & Aire limpio & Aplicación & B. & $\begin{array}{l}\text { ldentificación de los } \\
\text { servicios a distancias } \\
\text { caminables, } \\
\text { promoviendo el uso no } \\
\text { motorizado. } \\
\text { Conectividad no } \\
\text { motorizada. Escenario } \\
\text { base cumpliendo con } \\
75.44 \% \\
\begin{array}{l}\text { Conectividad r no } \\
\text { motorizada. Escenario } \\
\text { proyectado } \\
100 \%\end{array}\end{array}$ & $\begin{array}{lr}\text { Por cada } & 100 \\
\text { km, reducir } & 174 \\
\text { CO2 kg de } \\
\text { CO2e }\end{array}$ & $\begin{array}{l}75.44 \% \text { de lo } \\
\text { esperado }\end{array}$ & $\begin{array}{l}50 \% \text { de lo } \\
\text { esperado }\end{array}$ \\
\hline
\end{tabular}




\begin{tabular}{|c|c|c|c|c|c|c|c|}
\hline Calidad urbana & Aire limpio & Aplicación & C. & $\begin{array}{l}\text { Inserción del conjunto } \\
\text { Habitacional a la } \\
\text { mancha urbana } \\
\text { Escenario base= } 100 \% \\
\text { de integración con la } \\
\text { acción urbanística } \\
\text { Escenario proyectado= } \\
100 \% \text { de integración } \\
\text { con la acción } \\
\text { urbanística }\end{array}$ & $\begin{array}{l}3 \text { árboles } \\
\text { mínimo por } \\
\text { habitante o } \\
9 \mathrm{~m} 2 \text { mínimos } \\
\text { de áreas } \\
\text { verdes por } \\
\text { cada habitante } \\
9 \mathrm{~m} 2 \text { de área } \\
\text { verde o bien } 1 \\
\text { árbol= absorbe } \\
\text { aprox. } 11.79 \mathrm{~kg} \\
\text { de dióxido de } \\
\text { carbono (OMS) }\end{array}$ & $\begin{array}{l}100 \% \text { de } \\
\text { inserción a } \\
\text { la acción } \\
\text { urbanística } \\
4.73 \mathrm{~m} 2 \text { de } \\
\text { áreas verde } \\
\text { aprox. } 50 \% \\
\text { de lo } \\
\text { esperado }\end{array}$ & $\begin{array}{l}900 \text { árboles } \\
2.7 \% \text { de lo } \\
\text { esperado }\end{array}$ \\
\hline
\end{tabular}

Tabla 4: Con el nivel metodológico 1. Elaboración propia

\begin{tabular}{|c|c|c|}
\hline Tipo & Elemento del dato & Método de recolección de datos \\
\hline 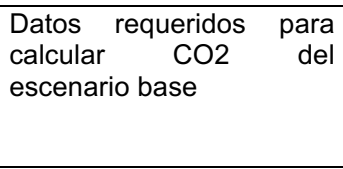 & $\begin{array}{l}\text { Identificación de los servicios de TP con sus } \\
\text { respectivas distancias en los recorridos en } \\
\mathrm{km} \text {. }\end{array}$ & $\begin{array}{l}\text { Para Guadalajara: } \\
\text { Estudio previo (Córdova, 2015) ()., google maps, Rutas } \\
\text { GDL. } \\
\text { Para Curitiba: } \\
\text { Estudio previo Fortunato, 2014., google maps, urbs. }\end{array}$ \\
\hline $\begin{array}{lcr}\text { Datos } & \text { requeridos } & \text { para } \\
\text { calcular } & \mathrm{CO} 2 & \text { del } \\
\text { escenario proyectado }\end{array}$ & $\begin{array}{l}\text { Identificación de los servicios de TP con sus } \\
\text { respectivas distancias en los recorridos en } \\
\mathrm{km} \text {. }\end{array}$ & $\begin{array}{l}\text { Para ambas: } \\
\text { Estimación con base a la calculadora de carbono } \\
\text { (Iniciativa Verde, 2016). }\end{array}$ \\
\hline 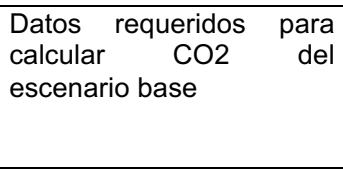 & $\begin{array}{l}\text { Identificación de los servicios básicos con } \\
\text { sus respectivas distancias de proximidad en } \\
\mathrm{km} \text {. }\end{array}$ & $\begin{array}{l}\text { Para Guadalajara: } \\
\text { Estudio previo (Córdova, 2015) ()., google maps, Rutas } \\
\text { GDL. } \\
\text { Para Curitiba: } \\
\text { Estudio previo Fortunato, 2014., google maps, urbs.. }\end{array}$ \\
\hline $\begin{array}{lcr}\text { Datos } & \text { requeridos } & \text { para } \\
\text { calcular } & \mathrm{CO} 2 & \text { del } \\
\text { escenario proyectado } & \end{array}$ & $\begin{array}{l}\text { Identificación de los servicios básicos con } \\
\text { sus respectivas distancias de proximidad en } \\
\mathrm{km} \text {. }\end{array}$ & $\begin{array}{l}\text { Para ambas: } \\
\text { Estimación con base a la calculadora de carbono } \\
\text { (Iniciativa Verde, 2016). }\end{array}$ \\
\hline $\begin{array}{l}\text { Datos requeridos para } \\
\text { calcular la absorción del } \\
\text { CO2 del escenario base }\end{array}$ & $\begin{array}{l}\text { Número de árboles con relación a las } \\
\text { personas del conjunto habitacional, así como } \\
\text { área total del terreno. }\end{array}$ & $\begin{array}{l}\text { Para Guadalajara: } \\
\text { Estudio previo (Córdova, 2015) } \\
\text { Para Curitiba: } \\
\text { Estudio previo Fortunato, } 2014 .\end{array}$ \\
\hline $\begin{array}{l}\text { Datos requeridos para } \\
\text { calcular la absorción del } \\
\mathrm{CO} 2 \text { del escenario } \\
\text { proyectado }\end{array}$ & $\begin{array}{l}\text { Número de árboles con relación a las } \\
\text { personas del conjunto hab. Equivalencia de } \\
\text { la absorción de dióxido de carbono por árbol } \\
\text { en relación a un hab. }\end{array}$ & $\begin{array}{l}\text { Estimación con base al promedio establecido por la OMS } \\
\text { de } 9 \mathrm{~m} 2 \text { mínimo de áreas verde por } 1 \text { habitante en relación } \\
\text { a la proyección del caso de estudio. } \\
\text { Estimación con base al promedio establecido por la OMS } \\
\text { de } 3 \text { árboles por } 1 \text { habitante. }\end{array}$ \\
\hline
\end{tabular}

Tabla 5: Con el nivel metodológico 2. Elaboración propia

Para el cálculo de la evaluación nivel 2 se ha tomado el nivel 1 y 2 debido al estudio previo seleccionado algunos datos obtenidos son a pesar de la aplicación a través de un sistema de medición de sustentabilidad para los niveles 2 y sobre todo el 3 es necesario un mayor grado de especificidad en la colecta de datos, es por esto que en este estudio se hace el cálculo en base a estos dos niveles y poder comparar y ponderar el resultado final, la ecuación con la que se medirá el nivel 2 fue construida en términos de teoría de conjuntos donde se aludirá a obtener los resultados a través del "escenario base y proyectado".

En el algoritmo general de medición lo que se estará estimando a través de este cálculo es el potencial de reducción de emisiones de en $\mathrm{kg}$ de $\mathrm{CO} 2 \mathrm{e}$ a través de las categorías previas seleccionadas, en donde la reducción de emisiones será igual al escenario base menos el escenario proyectado en kilogramos de gas de efecto invernadero como se expresa en ecuación (1)

$$
\text { Reducción de emisiones }=\frac{\text { escenario base }}{\text { potencial de reducción en }} \frac{\text { escenario proyectado }}{\mathrm{kg} \text { de } \mathrm{CO}}
$$

Tomando así cada una de las estrategias a evaluar se arrojan los siguientes resultados, visualizados a través de la captura de los datos por medio de la tabla 6. 


\begin{tabular}{|c|c|c|c|c|c|c|c|c|c|}
\hline \multicolumn{2}{|c|}{$\begin{array}{l}\text { Categoría: Calidad } \\
\text { Urbana }\end{array}$} & \multirow{2}{*}{$\begin{array}{l}\text { Co- } \\
\text { beneficio } \\
\text { específico }\end{array}$} & \multirow{2}{*}{$\begin{array}{l}\text { Variable } \\
\text { estratégica } \\
\text { de } \\
\text { evaluación }\end{array}$} & \multirow{2}{*}{$\begin{array}{l}\text { Indicador de } \\
\text { evaluación } \\
\text { seleccionado }\end{array}$} & \multicolumn{3}{|c|}{$\begin{array}{l}\text { Selección de nivel de } \\
\text { metodología }\end{array}$} & \multirow{2}{*}{$\begin{array}{lr}\text { Resultado } & \text { caso } \\
\text { Guadalajara. } & \\
\text { Potencial } & \text { de } \\
\text { reducción } & \text { de } \\
\text { emisiones } & \end{array}$} & \multirow{2}{*}{$\begin{array}{lr}\text { Resultado } & \text { caso } \\
\text { Curitiba. } & \\
\text { Potencial } & \text { de } \\
\text { reducción } & \text { de } \\
\text { emisiones } & \end{array}$} \\
\hline $\begin{array}{l}\text { Categoría } \\
\text { Principal }\end{array}$ & $\begin{array}{l}\text { Sub- } \\
\text { categoría }\end{array}$ & & & & $\begin{array}{l}\text { Nivel } \\
1\end{array}$ & $\begin{array}{l}\text { Nivel } \\
2\end{array}$ & $\begin{array}{l}\text { Nivel } \\
3\end{array}$ & & \\
\hline \multirow[t]{3}{*}{$\begin{array}{l}\text { I.Aire } \\
\text { limpio }\end{array}$} & $\begin{array}{l}\text { Social/ } \\
\text { ambiental }\end{array}$ & $A$ & $\begin{array}{l}\text { Transporte } \\
\text { Público }\end{array}$ & $\begin{array}{l}\mathrm{kgCO} 2 \mathrm{e} \\
\text { a cada } 1000 \mathrm{~km} \\
\text { de recorrido }\end{array}$ & $x$ & $X$ & & $\begin{array}{l}\text { Nivel } 1 \\
25 \% \text { cumplimiento } \\
\text { nivel } 2 \text { escenario } \\
\text { base } \\
108,430 \mathrm{~kg} \text { de } \mathrm{CO} 2 \mathrm{e} \\
\text { Nivel } 2 \text { escenario } \\
\text { proyectado } \\
68,390 \mathrm{kgCO} 2 \mathrm{e}\end{array}$ & $\begin{array}{l}\text { Nivel } 1 \\
50 \% \\
\text { cumplimiento } \\
\text { nivel } 2 \text { escenario } \\
\text { base } \\
15,075,800 \mathrm{~kg} \text { de } \\
\text { CO2e } \\
\text { Nivel } 2 \text { escenario } \\
\text { proyectado } \\
10,844,700 \mathrm{~kg} \text { de } \\
\text { CO2e }\end{array}$ \\
\hline & $\begin{array}{l}\text { Social/ } \\
\text { ambiental }\end{array}$ & $B$ & $\begin{array}{l}\text { Distancias } \\
\text { de } \\
\text { proximidad }\end{array}$ & $\begin{array}{l}\mathrm{kgCO} 2 \mathrm{e} \\
\text { a cada } 100 \mathrm{~km} \\
\text { de recorrido }\end{array}$ & $x$ & $X$ & & $\begin{array}{l}\text { Nivel } 1 \\
75 \% \text { cumplimiento } \\
\text { Nivel } 2 \text { escenario } \\
\text { base } \\
2,695 \mathrm{kgCO} 2 \mathrm{e} \\
\text { potencial } \\
\text { reducción de } \\
\text { emisiones } \\
\text { Nivel 2 escenario } \\
\text { proyectado } \\
0\end{array}$ & $\begin{array}{l}\text { Nivel } 1 \\
50 \% \\
\text { cumplimiento } \\
\text { Nivel } 2 \text { escenario } \\
\text { base } \\
1,503,765 \\
\text { kgCO2e potencial } \\
\text { de reducción de } \\
\text { emisiones } \\
\text { Nivel } 2 \text { escenario } \\
\text { proyectado } \\
0\end{array}$ \\
\hline & Ambiental & C & $\begin{array}{l}\text { No. } \\
\text { Arboles }\end{array}$ & $\begin{array}{l}\text { Absorción de } \\
\text { kgCO2 } \\
9 \mathrm{~m} 2 \text { de área } \\
\text { verde por } \\
\text { persona }=35.37 \\
\text { kgCO2 } \\
\text { absorbidos } \\
\text { aprox. }\end{array}$ & $\mathrm{X}$ & $X$ & & $\begin{array}{l}\text { Nivel } 1 \\
50 \% \text { cumplimiento } \\
\text { Nivel } 2 \text { escenario } \\
\text { base } \\
10,575 \mathrm{kgCO} 2 \mathrm{e} \\
\text { absorbidos } \\
\text { Nivel } 2 \text { escenario } \\
\text { proyectado } \\
\begin{array}{l}11,708.1 \mathrm{~kg} \text { de } \mathrm{CO} 2 \mathrm{e} \\
\text { mínimo faltante de } \\
\text { absorción }\end{array}\end{array}$ & $\begin{array}{l}\text { Nivel } 1 \\
2.7 \% \\
\text { cumplimiento } \\
\text { Nivel } 2 \text { escenario } \\
\text { base 10,607 } \\
\text { kgCO2e } \\
\text { absorbidos } \\
\text { Nivel } 2 \text { escenario } \\
\text { proyectado } \\
381,996 \text { kg de } \\
\text { CO2e mínimo } \\
\text { faltante de } \\
\text { absorción para } \\
\text { los residentes } \\
\text { nuevos del } \\
\text { conjunto } \\
\text { habitacional. }\end{array}$ \\
\hline
\end{tabular}

Tabla 6: Tabla resumen de resultados obtenidos. Elaboración propia

\section{CONCLUSIONES}

En la tabla 6 de resultados obtenidos se hizo el vaciado de todos los ítems analizados tomando la localización e integración de la acción urbana; la conectividad no motorizada; así como la proximidad a las paradas de transporte público como la estrategia de sustentabilidad que es la base de la aplicación metodológica y su incidencia con la categoría uno de los co-beneficios en atención al Aire Limpio, en la parte de sub-categoría refiriéndose a esta dentro de la clasificación ambiental, social y económica de la sustentabilidad se encontraba el co-beneficio identificado en A, B, C, en el indicador de evaluación se tomó al transporte público, distancias de proximidad y metros cuadrados de áreas verdes, con lo cual se evaluarían a través de los recorridos, infraestructuras a los servicios y la vegetación en $\mathrm{kgCO} 2 \mathrm{e}$ emitidos, absorbidos o reducidos en base a los dos niveles de evaluación. Donde en la parte final se puede encontrar el resultado obtenido a través del nivel 1 (que fue dado por el porcentaje) luego del nivel 2 dado por la cantidad resultado del estudio a través de las ecuaciones, y en el que se hace referencia al escenario base a través del estudio previo por medio de las estrategias de sustentabilidad 
y al escenario proyectado al que de haber cumplido con el $100 \%$ del cumplimiento se abría referido y lo cual muestra un potencial de reducción en las mismas.

Con lo cual se localiza en primera instancia que es evidente el tener criterios de valor más cuantitativo para evitar las estimaciones por apreciación cualitativa, que insertan subjetividad a los objetivos obtenidos.

En segundo, en ambos casos se considera la planeación de forma tradicional deslocalizando los impactos inherentes en las estrategias previas adoptadas de forma que no se tiene una claridad en el resultado final a través de su manejo, o forma de interpretación en las lecturas de la ciudad de forma previsoria.

De igual manera en ambos casos basados en los estudios previos uno a través de la academia y el otro a través de una empresa, existe un brecho instrumentario a nivel metodológico lo cual inhibe la capacidad de poder implementar estrategas más adecuadas al contexto donde se implementarán para el cumplimiento de los objetivos prestablecidos a nivel local, regional, nacional y global lo que provoca que dichos parámetros sean muchas veces abandonados o bien solo parte de un discurso y no de una acción efectiva.

Ahora pues, si bien este estudio solo se analizan dos estrategias como punto de partida de la comprobación de los impactos ocultos o bien los co-beneficios inherentes en dos de las principales estrategias (movilidad y paisaje) encomendadas por la sustentabilidad como lo es la movilidad, la infraestructura a distancias de proximidad y las áreas verdes, que hace referencia a la estrategia de calidad urbana, se vio claro que no se ha podido superar la brecha dejada por este concepto, y con lo cual es posible viabilizar e identificar a través del enfoque de cobeneficios, de tal forma que fue posible localizar la incapacidad de la actual política de vivienda de interés social implementada en la ciudad de tomar en cuenta mecanismos que detecten de forma inmediata y cuantitativa los impactos ocultos de las estrategias implementadas en sus instrumentos reguladores en las dos ciudades objeto de estudio y a través de los mismos parámetros de evaluación, lo cual tiene como resultado una deslocalización difusa pero tangible en la ciudad.

Si bien para la correcta y profunda planeación de la ciudad, es posible y necesario abundar más con el análisis de otras estrategias a través de este enfoque de co-beneficios lo cual logre localizar a mayor alcance de forma efectiva y complementaria su poli-integración lo cual marque la dirección de las nuevas perspectivas de planeación a través de los co-beneficios en la vivienda de interés social facilitando así pues a los tomadores de decisiones la óptima ruta para el logro efectivo de la viabilización a través de los sistemas de planeación la creación y fomento de verdaderas ciudades sustentables.

\section{REFERENCIAS}

LEITE, C., \& MARQUES AWAD, J. D. (2014). Cidades sustentáveis, cidades inteligentes. Sao Paulo, Sao Paulo, Brasil: Bookman.

ACSELRAD, H., \& Leroy, J.-P. (s.f.). Novas Premissas da Sustentabilidade Democrática. Projeto Brasil Sustentável e Democrático. Brasil : RITS.

ABIKO. (1995). Introdução à Gestão Habitacional. Escola Politécnica da USP, Departamento de Engenharia de Construção Civil. Sao Paulo: USP.

BALABAN, O. (2013). Co-benefits of green buildings and the opportunities and barriers regarding their promotion (Vol. No. 171). Turquía: UNU-IAS y TUBITAK.

ESMANHOTTO C. Contexto y prospectiva de la vivienda social en Curitiba. [recopil.] Dulce Esmeralda García Ruiz. Curitiba, 28 de Octubre de 2016

REBELO V. Contexto de la Vivienda de Interés Social en Curitiba. [trad.] Dulce Esmeralda García Ruiz. [recopil.] Dulce Esmeralda García Ruiz. Curitiba, 28 de Octubre de 2016. 
FORTUNATO, R. (2014). A sustentabilidade na habitação de interesse social: Estudos de caso em reassentamentos do Programa Minha Casa, Minha Vida no Nucleo Central da Região etropolitana da Curitibamunicipoios de Curitiba e Fazeda Rio Grande. Universidade Federal do Paraná, Curitiba.

FOLADORI, G. y PIERRI, N. (2005). ¿sustentabilidad? desacuerdos sobre el desarrollo sustentable. Cd. de México, México: Universidad Autónoma de Zacatecas y Miguel Angel Porrúa.

GÁNDARA, G. y OSORIO, F. (2014). Métodos prospectivos: manual para el estudio y la construcción del futuro. México, D.F.: PAIDÓS.

GARCÍA, D. E. (2015). 1er simposio vivienda digna, sustentable y resiliente, (págs. 1-20). Guadalajara.

GARCÍA, D. E. (2016). (J. R. Cladera, Ed.) Barcelona: Centre of Land Policy and Valuations CPSV.

GARRIDO, L. (2012). Un Nuevo Paradigma en Arquitectura. Barcelona, España: Monsa.

HABITAT III. (2016). Proyecto de documento final de la Conferencia de las Naciones Unidas sobre la Vivienda y el Desarrollo Urbano Sostenible (Hábitat III). Quito: Naciones Unidas.

IMEPLAN. (2016). Plan de Ordenamiento Territorial Metropolitano del AMG. Instituto Metropolitano de Planeamiento. Area Metropolitana de Guadalajara: V.IIIFB-aprobacion Ayuntamientos.

IPPUC. (2015). "Revisão/2014. Plano Diretor de Curitiba. Linhas de proposta para o plano diretor" Curitiba: Prefeitura de Curitiba \& IPPUC.

Manual for Quantitative evaluation of the co-benefits approach to climate change projects (Vol. Version 1.0). (2009).

ROGERS, R., \& Gumuchdjian, P. (2015). Cidades para um pequeno planeta. (A. R. Marco, Trad.) Sao Paulo, Sao Paulo, Brasil: Gustavo Gili.

ROGUSKI, M. (2016). Panel I. Engajamento Comunitário, I Encontro de urbanismo colaborativo. Curitiba: COURB.

SMITH, A. (2013). The climate bonus. Co-benefit of Climate Policy. (Kindle, Ed.) Abingdon: Routledge y Earthscan.

THIELE, L. P. (2013). Sustainability, polity key concepts (Kindle ed.). Cambridge, UK: whiley.

\subsection{Fuentes electrónicas:}

CONAVI. (21 de Septiembre de 2016). gob.mx. Recuperado el 29 de Noviembre de 2016, de Programa de Acceso al Financiamiento para Soluciones Habitacionales: http://www.gob.mx/cms/uploads/attachment/file/140911/Presentacion_accesible_al_Programa_190916.pdf

CONAVI. (25 de julio de 2016). gob.mx. Recuperado el 29 de noviembre de 2016, de Comisión Nacional de Vivienda: https://www.gob.mx/conavi/acciones-y-programas/comision-nacional-de-vivienda?idiom=es

Banco Interamericano de Desarrollo. (2014). Guia metodológica iniciativa ciudades emergentes y sostenibles. Iniciativa Ciudades Emergentes y Sostenibles. Recuperado el 2016 de Noviembre de 13, de http://www.iadb.org/es/temas/ciudades-emergentes-y-sostenibles/publicaciones-ciudades-

sostenibles, 18715. html

INFONAVIT. (Abril de 2016). Demanda Potencial - Jalisco - INFONAVIT. Recuperado el 29 de Noviembre de 2016, de SNIIV2.0: http://sniiv.conavi.gob.mx/Reports/Infonavit/Demanda_Pot.aspx 
NAMA de Vivienda. (18 de abril de 2016). Documentos de NAMA de Vivienda Nueva. Recuperado el 29 de noviembre de 2016, de gob.mx: https://www.gob.mx/conavi/documentos/nama-de-vivienda-nueva28835?idiom $=$ es

NAMA de Vivienda Nueva. (13 de abril de 2016). NAMA de Vivienda Nueva. Recuperado el 29 de noviembre de 2016, de gob.mx: https://www.gob.mx/conavi/acciones-y-programas/nama-de-vivienda-nueva

NAMA de vivienda nueva. (13 de abril de 2016). Proyectos pilotos de la NAMA de vivienda nueva Guadalajara. Recuperado el 29 de noviembre de 2016, de gob.mx: https://www.gob.mx/cms/uploads/attachment/file/83250/NAMA VN GUADALAJARA.pdf

NAMA Mexicana de Vivienda Sustentable. (14 de abril de 2016). NAMA Mexicana de Vivienda Sustentable. Recuperado el 29 de noviembre de 2016, de gob.mx: https://www.gob.mx/conavi/acciones-y-programas/namamexicana-de-vivienda-sustentable

NAMA Mexicana de Vivienda Sustentable. (14 de abril de 2016). NAMA Mexicana de Vivienda Sustentable. Recuperado el 29 de noviembre de 2016, de gob.mx: https://www.gob.mx/conavi/acciones-y-programas/namamexicana-de-vivienda-sustentable

Ministério das Cidades. (24 de Marzo de 2016). Programa Minha Casa, Minha Vida. Ministério das cidades. Recuperado el 16 de Noviembre de 2016 de Ministerio das cidades: http://www.cidades.gov.br/habitacao-cidades/programa-minha-casaminha-vida-pmcmv.

ONU HABITAT. (2016). Por un mejor futuro urbano. Viviendas y mejoramiento de asentamientos precarios. Recuperado el 2016 de Noviembre de 13, de ONU HABITAT: http://es.unhabitat.org/temas-urbanos/viviendas/ 